\title{
Semi-embeddings and weakly sequential completeness of the projective tensor product
}

\author{
by
}

QINGYING BU (University, MS)

\begin{abstract}
We show that if $\left\{P_{k}\right\}$ is a boundedly complete, unconditional Schauder decomposition of a Banach space $X$, then $X$ is weakly sequentially complete whenever $P_{k} X$ is weakly sequentially complete for each $k \in \mathbb{N}$. Then through semi-embeddings, we give a new proof of Lewis's result: if one of Banach spaces $X$ and $Y$ has an unconditional basis, then $X \widehat{\otimes} Y$, the projective tensor product of $X$ and $Y$, is weakly sequentially complete whenever both $X$ and $Y$ are weakly sequentially complete.
\end{abstract}

1. Introduction. The semi-embeddings in Banach spaces were introduced by Lotz, Peck, and Porta (see [10]). A Banach space $X$ is said to semi-embed into a Banach space $Y$ if there is a one-to-one continuous linear operator from $X$ to $Y$ such that the image of the closed unit ball of $X$ is closed in $Y$. A Banach space property is said to be semi-embedding inherited if it is inherited from a Banach space $Y$ to a separable Banach space $X$ whenever $X$ semi-embeds into $Y$. Delbaen (see [1]) showed that the Radon-Nikodym property is semi-embedding inherited. Moreover, the near Radon-Nikodym property (see [7]), the analytic Radon-Nikodym property and a type I Radon-Nikodym property (see [3]), a type II Radon-Nikodym property (see [11]), the (analytic) complete continuity property, and a type II complete continuity property (see $[12,13]$ ) are semi-embedding inherited properties.

In addition, non-containment of $c_{0}$ is also a semi-embedding inherited property due to Dowling's result: a Banach space contains no copy of $c_{0}$ if and only if it has some type I Radon-Nikodym property (see [4]).

However, reflexivity and non-containment of $\ell_{1}$ are not semi-embedding inherited. For example, $\ell_{2} \widehat{\otimes} \ell_{2}$ semi-embeds into $\ell_{2}^{\text {strong }}\left(\ell_{2}\right)$ (see [2]). It is known that $\ell_{2}^{\text {strong }}\left(\ell_{2}\right)$ is reflexive and does not contain a copy of $\ell_{1}$. But $\ell_{2} \widehat{\otimes} \ell_{2}$ contains a complemented copy of $\ell_{1}$ (see [14, p. 23]).

Recently Bu, Diestel, Dowling, and Oja [2] showed that if $U$ is a Banach space with a boundedly complete, 1-unconditional basis and if $X$ is any

2000 Mathematics Subject Classification: Primary 46M05, 46B28, 46B22. 
Banach space, then $U \widehat{\otimes} X$, the projective tensor product of $U$ and $X$, semiembeds into an $X$-valued Banach sequence space $U(X)$. Then they used this semi-embedding to show that several types of Radon-Nikodym properties are inherited from Banach spaces (one of which has an unconditional basis) to their projective tensor product. In this paper, we will also use this same semi-embedding to show that the weakly sequential completeness is inherited from $U(X)$ to $U \widehat{\otimes} X$, and then use this inheritance to give a short proof of Lewis's result [8]: the weakly sequential completeness is inherited from Banach spaces (one of which has an unconditional basis) to their projective tensor product.

For any Banach space $X$, its topological dual and closed unit ball will be denoted by $X^{*}$ and $B_{X}$, respectively. For two Banach spaces $X$ and $Y$, let $X \widehat{\otimes} Y$ denote the completion of the tensor product $X \otimes Y$ with respect to the projective tensor norm; and let $\mathcal{L}(X, Y)$ denote the space of all continuous linear operators from $X$ to $Y$.

\section{Semi-embeddings}

Definition 1. A continuous linear operator $T$ from a Banach space $X$ to a Banach space $Y$ is called a semi-embedding if $T$ is one-to-one and $T\left(B_{X}\right)$ is closed in $Y$. A Banach space $X$ is said to semi-embed into a Banach space $Y$ if there is a semi-embedding from $X$ to $Y$.

Lemma 2. Suppose that $X$ and $Y$ are Banach spaces such that $Y$ is weakly sequentially complete and there is a semi-embedding $T$ from $X$ to $Y$. If $\left\{x_{n}\right\}_{n=1}^{\infty}$ is a weakly Cauchy sequence in $X$, then there exists an $x \in X$ such that $T\left(x_{n}\right)$ converges to $T(x)$ weakly in $Y$.

Proof. Since $T$ is weakly-weakly continuous, $\left\{T\left(x_{n}\right)\right\}_{n=1}^{\infty}$ is a weakly Cauchy sequence in $Y$. Thus there exists a $y \in Y$ such that $T\left(x_{n}\right)$ converges to $y$ weakly in $Y$. Let $z_{n}=x_{n} / c$, where $c=\sup _{n}\left\|x_{n}\right\|<\infty$. Then $z_{n} \in B_{X}$ and $T\left(z_{n}\right)$ converges to $y / c$ weakly in $Y$. Since $T\left(B_{X}\right)$ is closed and convex in $Y, T\left(B_{X}\right)$ is also weakly closed in $Y$. Thus $y / c \in T\left(B_{X}\right)$. Therefore there exists a $z \in B_{X}$ such that $T(z)=y / c$. Let $x=c z$. Then $x \in X$ and $T\left(x_{n}\right)$ converges to $T(x)=y$ weakly in $Y$.

Let $U$ be a Banach space with a boundedly complete, 1-unconditional basis. Let $\left\{e_{i}\right\}_{i=1}^{\infty}$ be a normalized, boundedly complete, unconditional basis of $U$ whose unconditional basis constant is 1 , and let $\left\{e_{i}^{*}\right\}_{i=1}^{\infty}$ be the normalized biorthogonal functionals associated to the basis $\left\{e_{i}\right\}_{i=1}^{\infty}$, i.e.,

$$
e_{i}^{*}\left(e_{j}\right)= \begin{cases}1 & \text { if } i=j \\ 0 & \text { if } i \neq j .\end{cases}
$$


For a Banach space $X$, define

$$
U(X)=\left\{\bar{x}=\left(x_{i}\right)_{i} \in X^{\mathbb{N}}: \sum_{i}\left\|x_{i}\right\| e_{i} \text { converges in } U\right\}
$$

and define a norm on $U(X)$ to be

$$
\|\bar{x}\|_{U(X)}=\left\|\sum_{i=1}^{\infty}\right\| x_{i}\left\|e_{i}\right\|_{U}
$$

Then $U(X)$ with this norm is a Banach space (see [2]). Moreover, by [2, Theorem 11], $U \widehat{\otimes} X$ semi-embeds into $U(X)$ through the semi-embedding $\psi$ defined as follows:

$$
\psi: U \widehat{\otimes} X \rightarrow U(X), \quad z \mapsto\left(\sum_{k=1}^{\infty} e_{i}^{*}\left(u_{k}\right) x_{k}\right)_{i},
$$

where $\sum_{k=1}^{\infty} u_{k} \otimes x_{k}$ is a representation of $z$.

Lemma 3. For each $T \in \mathcal{L}\left(U, X^{*}\right)$, define

$$
I_{T}: U \widehat{\otimes} X \rightarrow \ell_{1}, \quad z \mapsto\left(\left\langle\psi(z)_{i}, T e_{i}\right\rangle\right)_{i} .
$$

Then $I_{T}$ is a continuous linear operator.

Proof. For each $\varepsilon>0$, any element $z \in U \widehat{\otimes} X$ admits a representation $z=\sum_{k=1}^{\infty} u_{k} \otimes x_{k}$ such that

$$
\sum_{k=1}^{\infty}\left\|u_{k}\right\| \cdot\left\|x_{k}\right\| \leq\|z\|_{U \hat{\otimes} X}+\varepsilon .
$$

For each $\left(s_{i}\right)_{i} \in \ell_{\infty}$, define

$$
v_{k}=\sum_{i=1}^{\infty} s_{i} e_{i}^{*}\left(u_{k}\right) e_{i}, \quad k=1,2, \ldots
$$

Since $\left\{e_{i}\right\}$ is a 1-unconditional basis of $U$, it follows that $v_{k} \in U$ and

$$
\left\|v_{k}\right\| \leq\left\|\left(s_{i}\right)_{i}\right\|_{\ell_{\infty}} \cdot\left\|u_{k}\right\|, \quad k=1,2, \ldots
$$

Thus

$$
\begin{aligned}
\left|\sum_{i=1}^{\infty} s_{i}\left\langle\psi(z)_{i}, T e_{i}\right\rangle\right| & =\left|\sum_{i=1}^{\infty} s_{i}\left\langle\sum_{k=1}^{\infty} e_{i}^{*}\left(u_{k}\right) x_{k}, T e_{i}\right\rangle\right| \\
& =\left|\sum_{k=1}^{\infty}\left\langle x_{k}, \sum_{i=1}^{\infty} s_{i} e_{i}^{*}\left(u_{k}\right) T e_{i}\right\rangle\right|=\left|\sum_{k=1}^{\infty}\left\langle x_{k}, T v_{k}\right\rangle\right| \\
& =\left|\left\langle\sum_{k=1}^{\infty} v_{k} \otimes x_{k}, T\right\rangle\right| \leq\|T\| \cdot\left\|\sum_{k=1}^{\infty} v_{k} \otimes x_{k}\right\|_{U \hat{\otimes} X}
\end{aligned}
$$




$$
\begin{aligned}
& \leq\|T\| \cdot \sum_{k=1}^{\infty}\left\|v_{k}\right\| \cdot\left\|x_{k}\right\| \leq\|T\| \cdot \sum_{k=1}^{\infty}\left\|\left(s_{i}\right)_{i}\right\|_{\ell_{\infty}} \cdot\left\|u_{k}\right\| \cdot\left\|x_{k}\right\| \\
& \leq\|T\| \cdot\left\|\left(s_{i}\right)_{i}\right\|_{\ell_{\infty}} \cdot\left(\|z\|_{U \widehat{\otimes} X}+\varepsilon\right) .
\end{aligned}
$$

It follows that

$$
\left\|\left(\left\langle\psi(z)_{i}, T e_{i}\right\rangle\right)_{i}\right\|_{\ell_{1}} \leq\|T\| \cdot\left(\|z\|_{U \widehat{\otimes} X}+\varepsilon\right) .
$$

Letting $\varepsilon \rightarrow 0$ gives

$$
\left\|\left(\left\langle\psi(z)_{i}, T e_{i}\right\rangle\right)_{i}\right\|_{\ell_{1}} \leq\|T\| \cdot\|z\|_{U \widehat{\otimes} X} \cdot
$$

Therefore $I_{T}$ is well defined and continuous.

THEOREM 4. The weak sequential completeness is inherited from $U(X)$ to $U \widehat{\otimes} X$.

Proof. Suppose $U(X)$ is weakly sequentially complete. Let $\left\{z_{n}\right\}_{n=1}^{\infty}$ be a weakly Cauchy sequence in $U \widehat{\otimes} X$. Since $\psi$ defined in (1) is a semi-embedding from $U \widehat{\otimes} X$ to $U(X)$, by Lemma 2 , there exists a $z \in U \widehat{\otimes} X$ such that $\psi\left(z_{n}\right)$ converges to $\psi(z)$ weakly in $U(X)$. Next we will show that $z_{n}$ converges to $z$ weakly in $U \widehat{\otimes} X$.

Fix $T \in \mathcal{L}\left(U, X^{*}\right)=(U \widehat{\otimes} X)^{*}$. By Lemma 3, $I_{T}: U \widehat{\otimes} X \rightarrow \ell_{1}$ is continuous, and hence weakly-weakly continuous. Thus $\left\{I_{T}\left(z_{n}\right)\right\}_{n=1}^{\infty}$ is a weakly Cauchy sequence in $\ell_{1}$, and hence a relatively weakly sequentially compact subset of $\ell_{1}$. By the Schur property, $\left\{I_{T}\left(z_{n}\right)\right\}_{n=1}^{\infty}$ is a relatively sequentially compact subset of $\ell_{1}$. Therefore there exists, for each $\varepsilon>0$, an $m_{1} \in \mathbb{N}$ such that

$$
\sum_{i=m_{1}+1}^{\infty}\left|\left\langle\psi\left(z_{n}\right)_{i}, T e_{i}\right\rangle\right| \leq \varepsilon / 3, \quad n=1,2, \ldots
$$

Since $\left(\left\langle\psi(z)_{i}, T e_{i}\right\rangle\right)_{i} \in \ell_{1}$, there exists an $m_{2}>m_{1}$ such that

$$
\sum_{i=m_{2}+1}^{\infty}\left|\left\langle\psi(z)_{i}, T e_{i}\right\rangle\right| \leq \varepsilon / 3
$$

Note that $\psi\left(z_{n}\right)$ converges to $\psi(z)$ weakly in $U(X)$. It follows that $\psi\left(z_{n}\right)_{i}$ converges to $\psi(z)_{i}$ weakly in $X$ for each $i \in \mathbb{N}$. Thus there exists an $n_{0} \in \mathbb{N}$ such that for each $n>n_{0}$,

$$
\left|\left\langle\psi\left(z_{n}\right)_{i}-\psi(z)_{i}, T e_{i}\right\rangle\right|<\varepsilon / 3 m_{2}, \quad i=1, \ldots, m_{2} .
$$

Now let $z_{n}, z \in U \widehat{\otimes} X$ have representations

$$
z_{n}=\sum_{k=1}^{\infty} u_{k, n} \otimes x_{k, n}, \quad z=\sum_{k=1}^{\infty} u_{k} \otimes x_{k} .
$$


By (2)-(4), for each $n>n_{0}$,

$$
\begin{aligned}
&\left|\left\langle z_{n}-z, T\right\rangle\right|=\left|\sum_{k=1}^{\infty}\left\langle T u_{k, n}, x_{k, n}\right\rangle-\sum_{k=1}^{\infty}\left\langle T u_{k}, x_{k}\right\rangle\right| \\
&=\left|\sum_{k=1}^{\infty}\left\langle\sum_{i=1}^{\infty} e_{i}^{*}\left(u_{k, n}\right) T e_{i}, x_{k, n}\right\rangle-\sum_{k=1}^{\infty}\left\langle\sum_{i=1}^{\infty} e_{i}^{*}\left(u_{k}\right) T e_{i}, x_{k}\right\rangle\right| \\
&=\left|\sum_{i=1}^{\infty}\left\langle\sum_{k=1}^{\infty} e_{i}^{*}\left(u_{k, n}\right) x_{k, n}, T e_{i}\right\rangle-\sum_{i=1}^{\infty}\left\langle\sum_{k=1}^{\infty} e_{i}^{*}\left(u_{k}\right) x_{k}, T e_{i}\right\rangle\right| \\
&=\left|\sum_{i=1}^{\infty}\left\langle\psi\left(z_{n}\right)_{i}-\psi(z)_{i}, T e_{i}\right\rangle\right| \\
& \leq \sum_{i=1}^{m_{2}}\left|\left\langle\psi\left(z_{n}\right)_{i}-\psi(z)_{i}, T e_{i}\right\rangle\right| \\
&+\sum_{i=m_{2}+1}^{\infty}\left|\left\langle\psi\left(z_{n}\right)_{i}, T e_{i}\right\rangle\right|+\sum_{i=m_{2}+1}^{\infty}\left|\left\langle\psi(z)_{i}, T e_{i}\right\rangle\right| \\
& \leq \varepsilon .
\end{aligned}
$$

Therefore $z_{n}$ converges to $z$ weakly in $U \widehat{\otimes} X$, and hence $U \widehat{\otimes} X$ is weakly sequentially complete.

\section{Weak sequential completeness and Schauder decompositions.} Let $X$ be a Banach space. A Schauder decomposition of $X$ is a sequence $\left\{P_{k}\right\}$ of continuous projections on $X$ such that $P_{i} \circ P_{j}=0$ whenever $i \neq j$, and $x=$ $\sum_{k=1}^{\infty} P_{k} x$ for each $x$ in $X$ (see [6] or [9, §1.g]). A Schauder decomposition $\left\{P_{k}\right\}$ of $X$ is called boundedly complete if, whenever $\left\{\sum_{k=1}^{n} x_{k}\right\}_{n=1}^{\infty}$ is a bounded sequence with $x_{k} \in P_{k} X$ for each $k \in \mathbb{N}$, then $\sum_{k} x_{k}$ converges in $X$. A Schauder decomposition $\left\{P_{k}\right\}$ of $X$ is called unconditional if for each $x \in X$, the series $\sum_{k} P_{k} x$ converges to $x$ unconditionally. Let $K$ denote the unconditional constant of the unconditional Schauder decomposition $\left\{P_{k}\right\}$ of $X$. Then for each $x \in X$ and each sequence $\left\{\theta_{k}\right\}_{k=1}^{\infty}$ of signs,

$$
\left\|\sum_{k=1}^{\infty} \theta_{k} P_{k} x\right\| \leq K \cdot\left\|\sum_{k=1}^{\infty} P_{k} x\right\|=K \cdot\|x\| .
$$

Now for each $x^{*} \in X^{*}$, define

$$
I_{x^{*}}: X \rightarrow \ell_{1}, \quad x \mapsto\left(x^{*}\left(P_{k} x\right)\right)_{k}
$$

Since $\sum_{k} P_{k} x$ converges unconditionally, $\sum_{k} x^{*}\left(P_{k} x\right)$ converges unconditionally, and hence $\sum_{k=1}^{\infty}\left|x^{*}\left(P_{k} x\right)\right|<\infty$. Thus $I_{x^{*}}$ is well defined. Moreover, it follows from (5) that $\left\|I_{x^{*}}(x)\right\| \leq K \cdot\|x\| \cdot\left\|x^{*}\right\|$ for each $x \in X$. Therefore $I_{x^{*}}$ is continuous. 
TheOREM 5. Let $\left\{P_{k}\right\}$ be a boundedly complete, unconditional Schauder decomposition of a Banach space $X$. Then $X$ is weakly sequentially complete whenever $P_{k} X$ is weakly sequentially complete for each $k \in \mathbb{N}$.

Proof. Let $\left\{x^{(n)}\right\}_{n=1}^{\infty}$ be a weakly Cauchy sequence in $X$ and let $M=$ $\sup _{n}\left\|x^{(n)}\right\|$. Then $\left\{P_{k} x^{(n)}\right\}_{n=1}^{\infty}$ is a weakly Cauchy sequence in $P_{k} X$ for each $k \in \mathbb{N}$. Thus there exists, for each $k \in \mathbb{N}$, an $x_{k} \in P_{k} X$ such that

$$
\text { weak- } \lim _{n} P_{k} x^{(n)}=x_{k} \quad \text { in } P_{k} X, k=1,2, \ldots
$$

Each $x^{*} \in X^{*}$ can be considered as a member of $\left(P_{k} X\right)^{*}$ for each $k \in \mathbb{N}$ if it is restricted to $P_{k} X$. Now for each fixed $m \in \mathbb{N}$, there exists, from (7), an $n_{0} \in \mathbb{N}$ such that

$$
\left|x^{*}\left(P_{k} x^{\left(n_{0}\right)}-x_{k}\right)\right|<1 / m, \quad k=1, \ldots, m .
$$

It follows from (5) that

$$
\begin{aligned}
\left|x^{*}\left(\sum_{k=1}^{m} x_{k}\right)\right| & \leq\left|\sum_{k=1}^{m} x^{*}\left(P_{k} x^{\left(n_{0}\right)}-x_{k}\right)\right|+\left|\sum_{k=1}^{m} x^{*}\left(P_{k} x^{\left(n_{0}\right)}\right)\right| \\
& \leq 1+\left\|x^{*}\right\| \cdot\left\|\sum_{k=1}^{m} P_{k} x^{\left(n_{0}\right)}\right\| \\
& \leq 1+K\left\|x^{*}\right\| \cdot\left\|x^{\left(n_{0}\right)}\right\| \leq 1+K M\left\|x^{*}\right\| .
\end{aligned}
$$

Thus $\left\{\sum_{k=1}^{m} x_{k}\right\}_{m=1}^{\infty}$ is bounded. Therefore there exists an $x \in X$ such that $x=\sum_{k=1}^{\infty} x_{k}$. Next we want to show that $x^{(n)}$ converges to $x$ weakly in $X$.

For each fixed $x^{*} \in X^{*}, I_{x^{*}}$ defined in (6) is continuous, and hence weakly-weakly continuous. Since $\left\{x^{(n)}\right\}_{n=1}^{\infty}$ is a weakly Cauchy sequence in $X,\left\{I_{x^{*}}\left(x^{(n)}\right)\right\}_{n=1}^{\infty}$ is a weakly Cauchy sequence in $\ell_{1}$, and hence relatively weakly sequentially compact. By the Schur property, $\left\{I_{x^{*}}\left(x^{(n)}\right)\right\}_{n=1}^{\infty}$ is a relatively sequentially compact subset of $\ell_{1}$. Thus for each $\varepsilon>0$, there exists an $m_{1} \in \mathbb{N}$ such that

$$
\sum_{k=m_{1}+1}^{\infty}\left|x^{*}\left(P_{k} x^{(n)}\right)\right| \leq \varepsilon / 3, \quad n=1,2, \ldots
$$

Since $x=\sum_{k=1}^{\infty} x_{k}$, there exists an $m_{2}>m_{1}$ such that

$$
\left|\sum_{k=m_{2}+1}^{\infty} x^{*}\left(x_{k}\right)\right| \leq \varepsilon / 3 .
$$

By (7) there exists an $n_{0} \in \mathbb{N}$ such that for each $n>n_{0}$,

$$
\left|x^{*}\left(P_{k} x^{(n)}-x_{k}\right)\right|<\varepsilon / 3, \quad k=1, \ldots, m_{2} .
$$


It follows from (8)-(10) that for each $n>n_{0}$,

$$
\begin{aligned}
\left|x^{*}\left(x^{(n)}-x\right)\right|= & \left|\sum_{k=1}^{\infty} x^{*}\left(P_{k} x^{(n)}-x_{k}\right)\right| \\
\leq & \sum_{k=1}^{m_{2}}\left|x^{*}\left(P_{k} x^{(n)}-x_{k}\right)\right| \\
& +\sum_{k=m_{2}+1}^{\infty}\left|x^{*}\left(P_{k} x^{(n)}\right)\right|+\left|\sum_{k=m_{2}+1}^{\infty} x^{*}\left(x_{k}\right)\right| \\
\leq & \varepsilon .
\end{aligned}
$$

Therefore $x^{(n)}$ converges to $x$ weakly in $X$, and hence $X$ is weakly sequentially complete.

4. Short proof of Lewis's result. For each $k \in \mathbb{N}$, define

$$
P_{k}: U(X) \rightarrow U(X), \quad \bar{x} \mapsto\left(0, \ldots, 0, \stackrel{(k)}{x}_{k}, 0,0, \ldots\right) .
$$

Then by [2, Proposition 5], $\left\{P_{k}\right\}$ is a boundedly complete, unconditional Schauder decomposition of $U(X)$. Hence Theorem 5 yields the following.

Corollary 6. $U(X)$ is weakly sequentially complete if $X$ is weakly sequentially complete.

Finally, by using the results obtained above, we will give a short proof of the following result of Lewis [8].

TheOREm 7. Let $X$ and $Y$ be Banach spaces such that one of them has an unconditional basis. Then $X \widehat{\otimes} Y$, the projective tensor product of $X$ and $Y$, is weakly sequentially complete whenever $X$ and $Y$ are weakly sequentially complete.

Proof. Suppose that $X$ has an unconditional basis. Since $X$ is weakly sequentially complete, $X$ does not contain a copy of $c_{0}$. By James's result [5] (or see [9, Theorem 1.c.10]), $X$ has a boundedly complete unconditional basis. Without loss of generality, we can assume that $X$ has a boundedly complete, 1-unconditional basis. It follows immediately from Theorem 4 and Corollary 6 that $X \widehat{\otimes} Y$ is weakly sequentially complete.

\section{References}

[1] J. Bourgain and H. P. Rosenthal, Applications of the theory of semi-embeddings to Banach space theory, J. Funct. Anal. 52 (1983), 149-188.

[2] Q. Bu, J. Diestel, P. N. Dowling, and E. Oja, Types of Radon-Nikodym properties for the projective tensor product of Banach spaces, Illinois J. Math. 47 (2003), 13031326 . 
[3] P. N. Dowling, Radon-Nikodým properties associated with subsets of countable discrete abelian groups, Trans. Amer. Math. Soc. 327 (1991), 879-890.

[4] -, Duality in some vector-valued function spaces, Rocky Mountain J. Math. 22 (1992), 511-518.

[5] R. C. James, Bases and reflexivity of Banach spaces, Ann. of Math. 52 (1950), $518-527$.

[6] N. Kalton, Schauder decompositions and completeness, Bull. London Math. Soc. 2 (1970), 34-36.

[7] R. Kaufman, M. Petrakis, L. Riddle, and J. J. Uhl, Nearly representable operators, Trans. Amer. Math. Soc. 312 (1989), 315-333.

[8] D. R. Lewis, Duals of tensor products, in: Lecture Notes in Math. 604, Springer, 1977, 57-66.

[9] J. Lindenstrauss and L. Tzafriri, Classical Banach Spaces I. Sequence Spaces, Springer, 1977.

[10] H. P. Lotz, N. T. Peck, and H. Porta, Semi-embeddings of Banach spaces, Proc. Edinburgh Math. Soc. 22 (1979), 233-240.

[11] N. Randrianantoanina and E. Saab, Stability of some types of Radon-Nikodým properties, Illinois J. Math. 39 (1995), 416-430.

[12] M. Robdera and P. Saab, The analytic complete continuity property, J. Math. Anal. Appl. 252 (2000), 967-979.

[13] - - - Complete continuity properties of Banach spaces associated with subsets of a discrete abelian group, Glasgow Math. J. 43 (2001), 185-198.

[14] R. A. Ryan, Introduction to Tensor Products of Banach Spaces, Springer, 2002.

Department of Mathematics

University of Mississippi

University, MS 38677, U.S.A.

E-mail: qbu@olemiss.edu

Received July 23, 2004

Revised version February 11, 2005 